\title{
VLA OBSERVATIONS OF ORBITAL MOTIONS IN YLW 15
}

\author{
S. Curiel, ${ }^{1}$ J. M. Girart, ${ }^{2}$ L. F. Rodríguez, ${ }^{3}$ and J. Cantó ${ }^{1}$ \\ RESUMEN
}

Reportamos la detección de movimientos propios en las componentes de la fuente binaria YLW 15, mediante observaciones de alta resolución angular $\left(\sim 00^{\prime \prime} 3\right)$ en el VLA. Encontramos movimientos propios absolutos del orden de $15 \mathrm{~km} \mathrm{~s}^{-1}$. La astrometría relativa entre ambas componentes revela movimientos orbitales que sugieren que la masa total del sistema es de $\sim 1.7 M_{\odot}$, y que VLA 1 tiene una masa mayor que VLA 2.

\section{ABSTRACT}

Using high angular resolution $\left(\sim 00^{\prime \prime} 3\right)$ Very Large Array (VLA) observations made at $3.6 \mathrm{~cm}$ during the period 1990 to 2002 , we report the detection of proper motions in the components of the binary source YLW 15 . We find absolute proper motions of the order of $15 \mathrm{~km} \mathrm{~s}^{-1}$. In addition, the relative astrometry between the two components reveals orbital proper motions that suggest that the total mass of this binary system is $\sim 1.7 M_{\odot}$, and that VLA 1 is more massive than VLA 2.

\section{Key Words: ISM: JETS AND OUTFLOWS - STARS: INDIVIDUAL (YLW 15) - STARS: PRE-MAIN SEQUENCE}

\section{INTRODUCTION}

YLW 15 (IRS 43) is an infrared protostar with a bolometric luminosity of $\sim 10 \mathrm{~L}_{\odot}$ (Young, Lada \& Wilking 1986; Wilking, Lada \& Young 1989) located in L1681B, a small cloud in the $\rho$ Ophiuchus molecular complex. It has been classified as a very young, class I object by André, Ward-Thompson \& Barsony (1993) on the basis of its infrared spectral energy distribution and its association with a compact bipolar outflow (Bontemps et al. 1996). YLW 15 has been proposed to be representative of the youngest lowmass stars detectable in X-rays (Grosso et al. 1997). Recent high angular resolution radio continuum observations (Girart, Rodríguez \& Curiel 2000; Girart et al. 2004) have revealed that this source is in fact a subarcsecond $\left(0{ }^{\prime \prime} 6\right)$ radio binary, with both components (VLA 1 and VLA 2) spatially extended.

Here, we analyze VLA observations made at $3.6 \mathrm{~cm}$ in the A configuration at several epochs from 1990 to 2002 . These observations resolve the two components of the YLW 15 system, allowing both absolute and relative astrometry to be performed.

\section{2: RESULTS AND CONCLUSIONS}

In Figure 1, we show the images for the first and last observed epochs, where the two components

\footnotetext{
${ }^{1}$ Instituto de Astronomía, UNAM, México. email: scuriel@astroscu.unam.mx

${ }^{2}$ Departament d'Astronomia i Meteorologia, Universitat de Barcelona, Barcelona, Spain.

${ }^{3}$ Instituto de Astronomía, UNAM, Morelia México.
}

(VLA 1 and VLA 2) of YLW 15 are identified. The absolute proper motion of the two sources to the southwest is evident in this figure. We made a linear least-squares fit to the positions of both components,

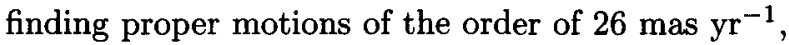
which, at a distance of $120 \mathrm{pc}$, imply a velocity in the plane of the sky of $\sim 15 \mathrm{~km} \mathrm{~s}^{-1}$. Very similar absolute proper motions have been found for the heavily obscured protostar in IRAS 16293-2422 (Loinard 2002) and for a group of three T Tauri stars (Teixeira et al. 2000; Loinard 2002), also in the $\rho$ Ophiuchus molecular cloud complex.

We have used the four epochs to perform relative astrometry, taking as reference the position of VLA 1. In Figure 1, we can see that the position angle of the vector that joins both sources seems to have changed slightly over the $11.8 \mathrm{yr}$ period between the observations. A conservative lower limit to the total mass of the system can be derived by assuming that the two components have circular motions around their mass center. In this case, in terms of the observed parameters, the mass of the system is given by $\left(M / \mathrm{M}_{\odot}\right) \geq 1.3\left(\mu / \mathrm{mas}_{\mathrm{yr}}^{-1}\right)^{2}(s / 100$ $\operatorname{mas})(d / \mathrm{kpc})^{3}$, where $\mu$ is the relative proper motion, $s$ is the angular separation between the members of the binary, and $d$ is the distance to the source. For $\mu=11.1 \operatorname{mas~yr}^{-1}, s=606$ mas, and $d=120 \mathrm{pc}$, we obtain $M \geq 1.7 \mathrm{M}_{\odot}$. If we further assume that the observed separation is close to the true separation, we can estimate an upper limit to the period of the system to be $P \simeq 360 \mathrm{yr}$. 
a

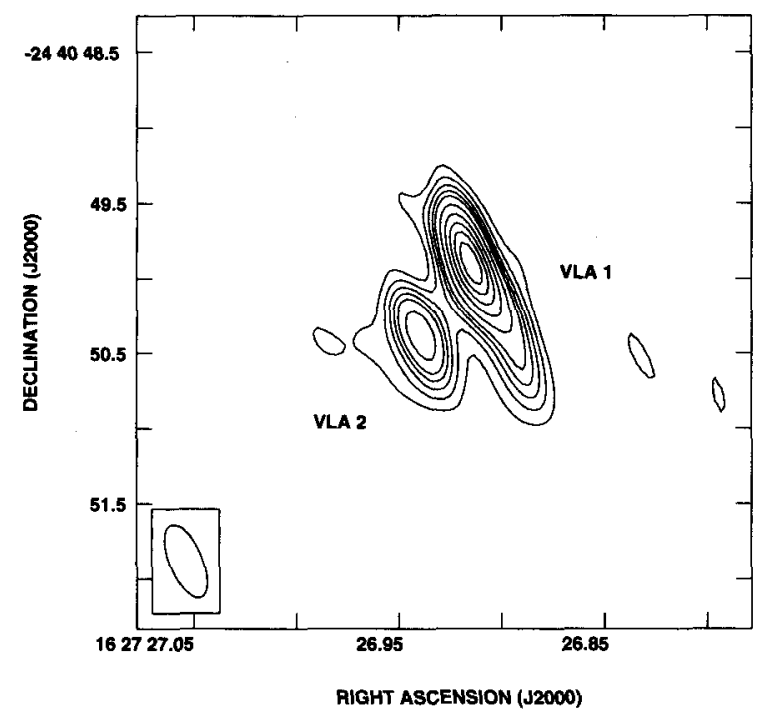

b

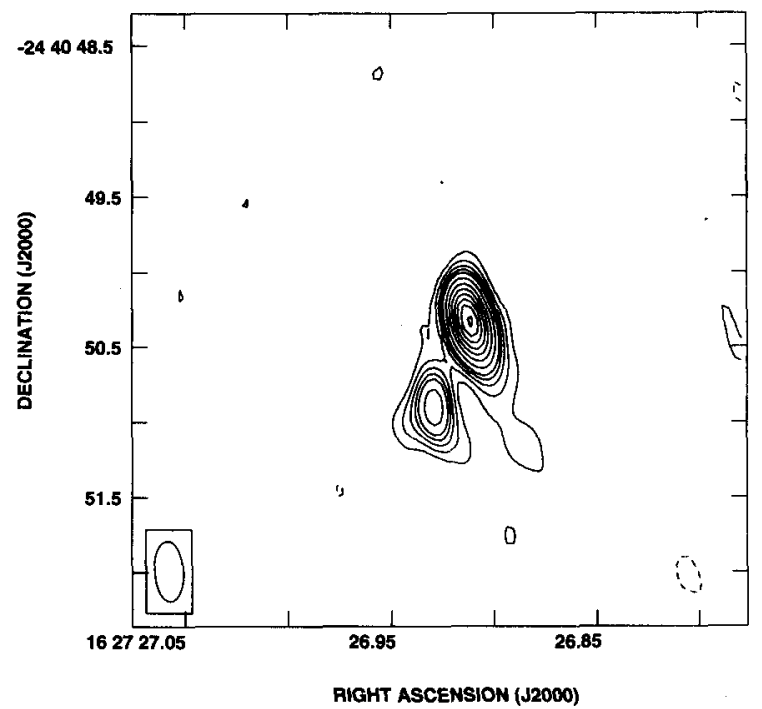

Fig. 1. VLA continuum images of YLW 15 at $3.6 \mathrm{~cm}$, for the epochs 1990.41 (left) and 2002.18 (right). Contours are $-5,-3,3,5,7,9,11,15, \ldots$, and $45 \times$ the rms noise of the images $\left(18 \mu \mathrm{Jy}\right.$ beam ${ }^{-1}$ for 1990.41 and $22 \mu \mathrm{Jy} \mathrm{beam}^{-1}$ for 2002.18). The half-power contours of the beams are shown in the bottom left corners. VLA 1 and VLA 2 are indicated. Note the large absolute proper motions between the two epochs.
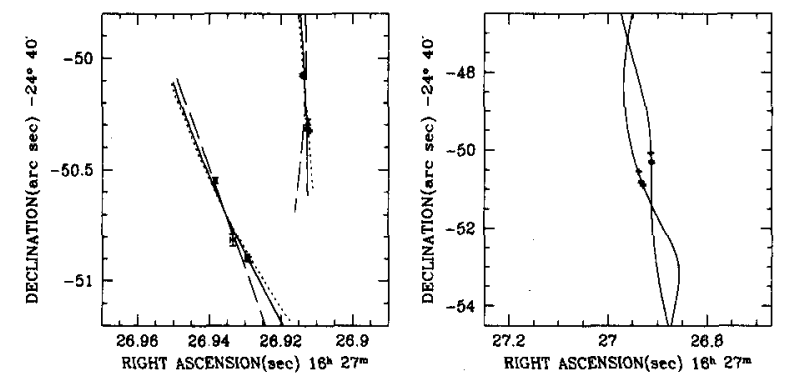

Fig. 2. Predicted proper motions for the YLW 15 binary system, assuming circular orbits on the plane of the sky. The left and right panels show the predicted orbits for an span of time of 40 years and of 500 years, respectively. The dotted lines correspond to the expected proper motions when assuming that VLA 1 is much more massive than VLA 2, the dashed lines when assuming that VLA 2 is much more massive than VLA 1 , and the solid lines when assuming a mass ratio of 2.4 between VLA 1 and VLA 2. Only solid lines are shown in the right panel.

We have fitted the absolute proper motions of both components by assuming that both sources are moving in circular orbits around their mass center, that the orbits are on the plane of the sky, and that the center of mass is moving in the plane of the sky. Figure 2 shows the predicted proper motions in a span of time of 40 years for the two limit cases $M_{V L A 1}>M_{V L A}$ and $M_{V L A 2}>>$ $M_{V L A 1}$, and the case in which $M_{V L A 1}=2.4 \times$ $M_{V L A}$. This figure shows that the observed proper motions of this binary system are better fitted if VLA 1 is much more massive than VLA 2. In fact, an excellent fit to the data can be obtained when assuming that the mass of VLA 1 and VLA 2 are about $1.2 \mathrm{M}_{\odot}$ and $0.5 \mathrm{M}_{\odot}$, respectively.

\section{REFERENCES}

André, P., Ward-Thompson, D., \& Barsony, M. 1993, ApJ, 406, 122

Bontemps, S., André, P., Terebey, S., \& Cabrit, S. 1996, A\&A, 311, 858

Girart, J. M., Rodríguez, L. F., \& Curiel, S. 2000, ApJ, $544, \mathrm{~L} 153$

Girart, J. M., Curiel, S., Rodríguez, L. F., \& Cantó, J. $2004, \mathrm{AJ}$, in press

Grosso, N., Montmerle, T., Feigelson, E. D., André, P., Casanova, S., \& Gregorio-Hetem, J. 1997, Nature, 387,56

Loinard, L. 2002, RevMexA\&A, 38, 61

Teixeira, R., Ducourant, C., Sartori, M. J., Camargo, J. I. B., Périé. J. P., Lépine, J. R. D., \& Benevides-Soares, P. 2000, A\&A, 361, 1143.

Young, E. T., Lada, C. J., \& Wilking, B. A. 1986, ApJ, $304, \mathrm{~L} 45$

Wilking, B. A., Lada, C. J., \& Young, E. T. 1989, ApJ, 340,823 